\title{
Thermal Analysis of Combustion Engine in Accordance to Temperature at the Outside Surfaces of the Engine
}

\author{
Zbigniew J. Sroka \\ Faculty of Mechanical Engineering, Wroclaw University of Technology, Wrocław, Poland \\ Email: zbigniew.sroka@pwr.edu.pl
}

Received 24 June 2014; revised 28 July 2014; accepted 30 August 2014

Copyright (C) 2014 by author and OALib.

This work is licensed under the Creative Commons Attribution International License (CC BY). http://creativecommons.org/licenses/by/4.0/

(c) (i) Open Access

\section{Abstract}

The internal combustion engine needs to be assessed in terms of thermal loads that come from different sources. Thermal analysis is important in both the design and the evaluation of the conditions of the engine. Interesting good results are obtained by association studies using non-invasive methods such as Infrared and mathematical modelling based on the boundary conditions identified in the experiment. The paper presents a methodology for measuring the surface temperature fields outside of the engine and the mathematical evaluation. The collected material will in future give opportunity to study dynamic thermal states based on the technique of reverse engineering.

\section{Keywords}

Combustion Engine, Thermo Vision, Models

Subject Areas: Analytical Chemistry, Physical Chemistry

\section{Introduction}

One of the trends in combustion engine development is to increase the power-to-displacement ratio by downsizing. The technical concept of engine downsizing is reduction of swept volume with support of other technologies like: direct fuel injection, variable valve timing, variable compression ratio and of course charging (turbo and/or compressor). It is very important that useful power has to be similar or even higher like the base engine [1]-[4].

Looking back to the year 1867, when first engine, called Otto I appeared, its specific power equalled 0.025 $\mathrm{kW} / \mathrm{dm}^{3}$ as result of volume $80 \mathrm{dm}^{3}$ giving power of $2 \mathrm{~kW}$. In 2012, small engine with $0.999 \mathrm{dm}^{3}$, manufactured by the Ford company as Eco Boost delivered power about $90.5 \mathrm{~kW}$-giving $90.60 \mathrm{~kW} / \mathrm{dm}^{3}$ of specific power ra- 
tio. Designers of that engine took care to meet the already very restrictive carbon dioxide emissions standards that will come into force in 2015 and above [5]-[10].

Combustion engine is loaded by heat, which comes from many simultaneous processes: the combustion itself, the friction, the fluid turnover (oil, fuel or coolant) and the ambient conditions. Application of downsizing gives problem of the heat flux from the engine to the atmosphere increasing climate warming. That is why one of the challenges when designing the engine will be to reduce thermal load of engine components by cooling systems, specific geometry design [11]-[13] etc.

The identification of the heat phenomenon mechanisms can be performed by mathematical models or via experiment. Very often both of them are employed, when thermal analysis made by theory is supported by boundary conditions taken from tests as in this project.

\section{Methodology and Test Bed}

Diesel engine, licensed SW400 Leyland, was tested by dynamometer with full load injection performance in the laboratory belongs to the Motor Division of Motor Vehicles and Combustion Engine at the Wroclaw University of Poland (Figure 1).

That engine is a perfect unit to downsize because of huge displacement of $6.5 \mathrm{dm}^{3}$, only $82.3 \mathrm{~kW}$ of power and around $360 \mathrm{Nm}$ of torque, it is a natural aspirated engine without any electronic support.

The investigation goal was to determinate thermal load of engine by average temperature on the outside surface in many points. From many reasons i.e. ecology, durability, strength points of views, the heating and cooling of engine operation states are crucial. To fulfil this task, the thermography system was used because of: being indirect measurement method, giving possibility to measure in dynamic way and in parallel paths. It is quite accurate measurements and what is most important non-influencing [14].

Engine was tested with the highest operating factors for full load characteristic.

The Thermovision system applied in the experiments was NEC 2100 with Saei type of matrix FPA detector (76,800 pixels). Range of measurement from $20^{\circ} \mathrm{C}$ to $1500^{\circ} \mathrm{C}$ and sensibility $<0.1^{\circ} \mathrm{C}$. For protocol, in order to give sensible results and to evaluate the average value of the temperature, the specific 20 points were chosen as shown on the Figure 2.

The temperature for mentioned above 20 points were measured from the start (heating up) until the time when engine reached the equilibrium state at ambient temperature after cooling process.

\section{Results}

All the following results have been collected to the in-built acquisition unit and then analysed by typical computer software.

The Figure 3 shows all data collected during entire run of engine.

Variable temperature curve is typical for the heating and cooling processes [15]. First, the relatively rapid increase in temperature and then cooling takes long time. Between these two phases occurs classic "saddle temperature" when with the exception of the engine initially rapid temperature drops to just increase. This increase is the result of stop of coolant circuit and other extortions of cooling (e.g. fan). This moment is dangerous for reasons of durability of parts, especially for the head and injectors. At the design stage, is should be properly protected the engine from degradation [16].

The following figures illustrate examples of changes in temperature for heating and cooling phases-for the cylinder head (Figure 4 and Figure 5).

On this basis it is possible to evaluate the "temperature time constant" i.e. the time to reach operating temperature of coolant, which decides on fuel consumption and emissions of toxic substance of exhaust gases. In that case, this time was estimated around 18 minutes. It is the result of the medium power units, compared to 8 10 minutes in the other models.

It is also possible to create mathematical models to predict the temperature and thermal load of engine components.

Examples of mathematical models to evaluate temperature at the all points vs. time unit $(t)$ are as Table 1.

Models appeared in Table 1, can be used in reverse engineering to make thermal analysis. For example it could be consider the heat diffusion, which expresses the quantity of thermal energy passed between mediums divided with barriers. 


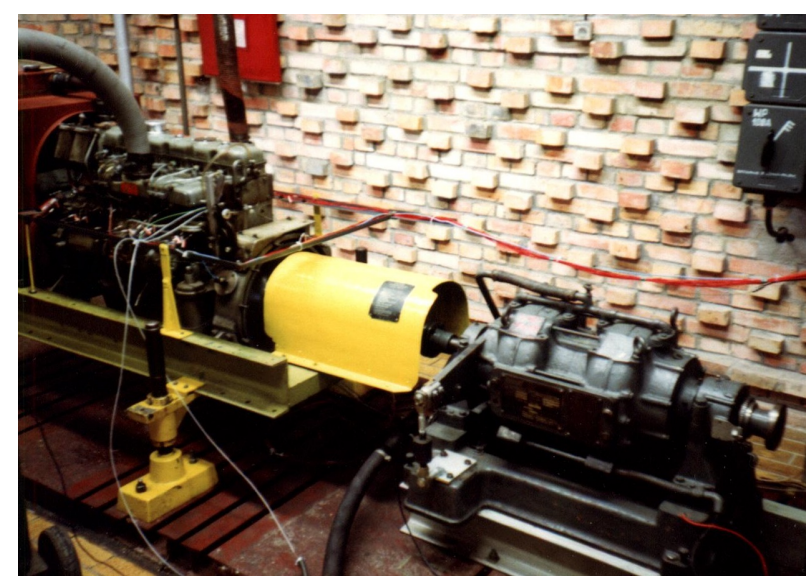

Figure 1. General view of the test bed in the laboratory.
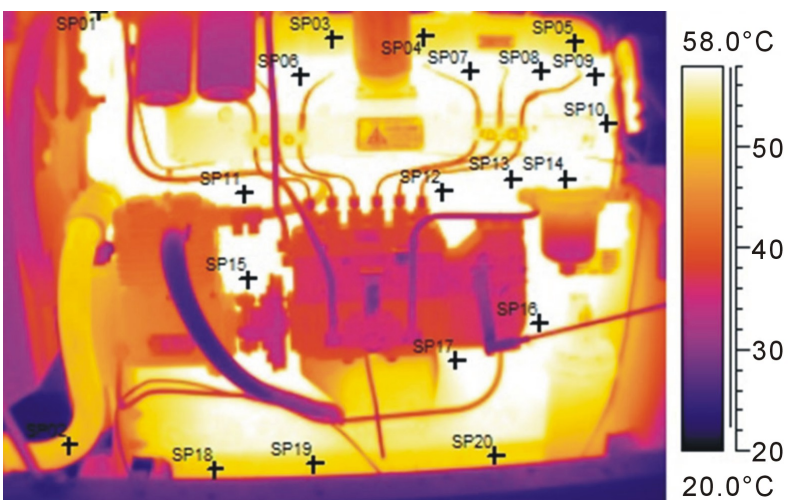

Figure 2. Thermogram with the layout of the samples performed on the SW400 engine.

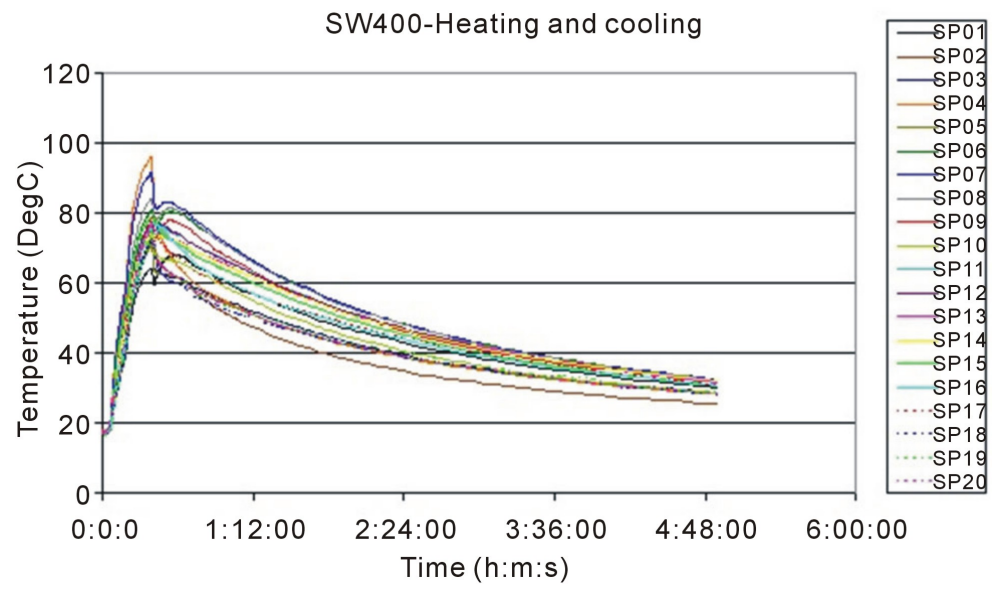

Figure 3. Temperature of sample points for heating and cooling process on SW400 engine.

For the unit dimensions of the studied parts of the engine the heat can be qualified according to the formula as shown below [15] [17] [18] (1):

$$
\mathrm{d} Q=c_{p} \cdot \rho \cdot \mathrm{d} x \cdot \mathrm{d} y \cdot \mathrm{d} z \cdot \frac{\partial T}{\partial t} \mathrm{~d} t
$$




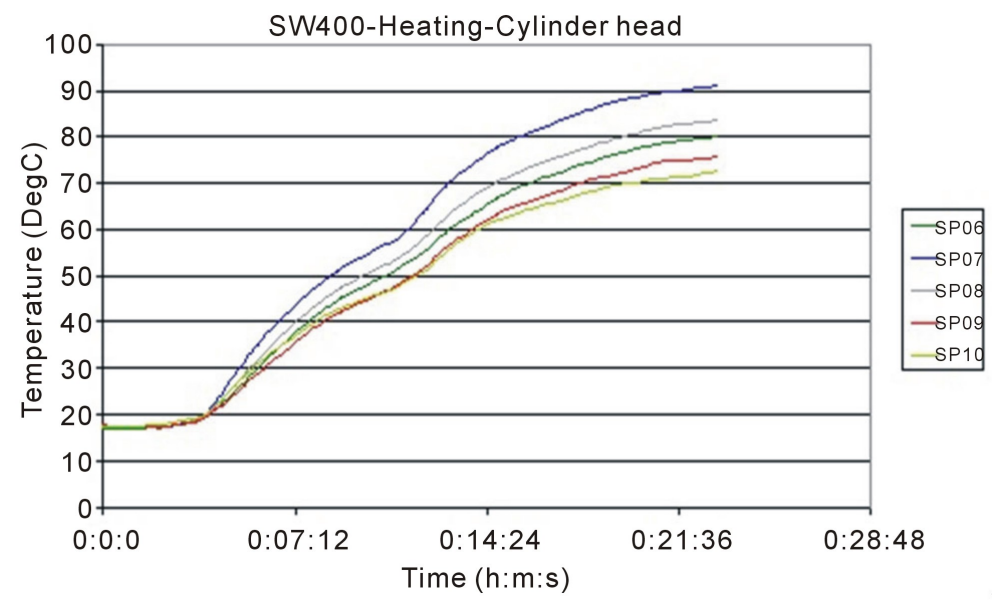

Figure 4. Heating process for the cylinder head.

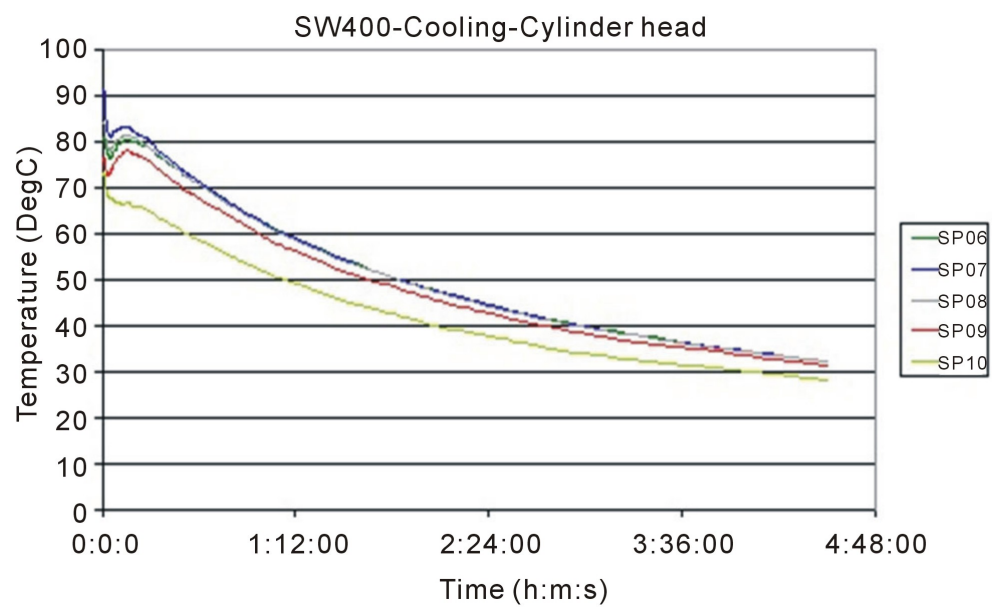

Figure 5. Cooling process for the cylinder head.

Table 1. Mathematical models for heating and cooling at chosen measuring points at engine surface (according to Figure 1).

\begin{tabular}{lccc}
\hline \multirow{2}{*}{ Point } & \multicolumn{1}{c}{ Heating phase } & \multicolumn{2}{c}{ Cooling phase } \\
\cline { 2 - 4 } & Model & $\mathrm{R}^{2}$ & Model \\
SP01 & $-61,620 t^{2}+4560+10$ & 0.98 & $66 \mathrm{e}^{-4.5 t}$ \\
SP05 & $2470 t^{2}+3993 t+11$ & 0.97 & $1248 t^{2}-417 t+65$ \\
SP07 & $-180,057 t^{2}+8534 t+5$ & 0.98 & $1612 t^{2}-574 t+85$ \\
SP12 & $-109,693 t^{2}+6322 t+8$ & 0.98 & $1180 t^{2}-458 t+77$ \\
SP17 & $-16,172 t^{2}+4680 t+10$ & 0.98 & $1717 t^{2}-546 t+76$ \\
SP20 & $-10,968 t^{2}+4440 t+11$ & 0.99 & $1614 t^{2}-493 t+68$ \\
\hline
\end{tabular}

SP01-test point on radiator, SP05—-test point on valve cover, SP07—-test point on cylinder head, SP12-test point on engine block (with coolant pass), SP17—-test point on engine block (without coolant pass), SP20 — test point on oil tank, $\mathrm{R}^{2}$ — correlation factor, $t$ - time unit.

where, $c_{p}$ —specific heat for constant pressure, $\rho$-density of material, $\mathrm{d} x, \mathrm{~d} y$, $\mathrm{d} z$-changes of unit dimensions of studied parts, $\mathrm{d} T$ - changes of temperature in the changes of time of $\mathrm{d} t$.

But, it is the next step in research.

\section{Conclusions}

The collected material shows clearly that the rate of heat absorption depends not only on the volume of each in- 
dividual item, but also on the temperature of neighbouring elements.

The warm-up is strongly dependent processes in the engine and the cooling phase is derived from the effects of the environment. For the options, the mathematical models were developed to predict thermal changes and their consequences in the form of thermal load of engine.

Diversity of factors for all mathematical models prevents building a single model for the whole engine-very useful when an engine is going to be downsized to recognize strong and weak points of construction of engine parts. Thermography appears to be a safe and friendly system for assessment of evolutionary changes in temperature for engine.

\section{References}

[1] Smith, A. (2008) Stroke of Genius for Gasoline Downsizing. Ricardo Q Rev, Q3.

[2] Sroka, Z.J. (2009) Micro Machines-The Concept of Engine Downsizing. Engine Technology International, 1, 58-62.

[3] Flierl, R., et al. (2011) Univalve—A Fully Variable Mechanical Valve Lift System for Future Internal Combustion Engines. Magazine ATZ, 4, 34-39.

[4] Merker, G.P., Schwartz, Ch. and Teichmann, R. (2009) Combustion Engines Development. Springer Edition.

[5] Bielaczyc, R. and Woodbrun, J. (2010) Global Trends in Emissions Regulation and Reduction. Combustion Engines Scientific Magazine, 142, 3-27.

[6] Shakun, J.D., et al. (2012) Global Warming Preceded by Increasing Carbon Dioxide Concentrations during the Last Deglaciation. Nature, 484, 49-54. http://dx.doi.org/10.1038/nature10915

[7] Regulation, E.C. (2009) nr 2009/33/WE of the European Parliament and of the Council—On the Promotion of Clean and Energy-Efficient Road Transport Vehicles. Brussels.

[8] Merkisz, J. and Radzimirski, S. (2007) Pollutant Emissions from Road Transportation in the Year 1970-2005 in Poland. Combustion Engines Scientific Magazine, 4-13.

[9] Tong, R.E. and Sanyol, A. (2012) Lower Energy Penalty $\mathrm{CO}_{2}$ Capture System. Carbon Capture Journal, 4.

[10] Kulazynski, M. and Sroka, Z.J. (2011) Developing Engine Technology. Print PAP, Lodz.

[11] Oppenheim, A.K., Kuhl, A.L., Packard, A.K., Hedrick, J.K. and Johnson, W.P. (1996) Model and Control of Heat Release in Engines. American Journal of Engine Combustion Flow Diagnosis, 1157, 15-23.

[12] Sroka, Z.J. (2012) Some Aspects of Thermal Load and Operating Indexes after Downsizing for Internal Combustion Engine. Journal of Thermal Analysis and Calorimetry, 110, 51-58. http://dx.doi.org/10.1007/s10973-011-2064-x

[13] Guzzella, L. and Onder, Ch.H. (2010) Introduction to Modelling and Control of Internal Combustion Engine Systems. 2nd Edition, Springer Editor. http://dx.doi.org/10.1007/978-3-642-10775-7

[14] Dudek, K. (1998) Thermography as a Diagnosing System. Journal of Transdisciplinary Systems Science, 3, 68-79.

[15] Heywood, J.B. (1989) Internal Combustion Engine Fundamentals. McGraw-Hill International Edition, Singapore.

[16] Sroka, Z.J. (2012) Thermal Load of Tuned Piston. Archives of Civil and Mechanical Engineering, 12, 342-347. http://dx.doi.org/10.1016/j.acme.2012.05.004

[17] Blair, G.P. (1996) Design and Simulation of Four-Stroke Engines. Edition SAE, Warrendale. http://dx.doi.org/10.4271/R-161

[18] Ramos, J.I. (1989) Internal Combustion Engine Modelling. American Publication Corporation, Hemisphere. 